\title{
Methodology for Selecting the Locaiton of an Optimum Viewing Point for an Ideal 3D Perspective View of a Given 3D Environment
}

\author{
${ }^{1}$ N. Sandya Rani and ${ }^{2}$ V. VaithyaNathan \\ ${ }^{1}$ MCA Department, RREC, Chennai - 95, Tamil Nadu, South India \\ ${ }^{2}$ Department of CSE, SASTRA, Tanjore, South India
}

\begin{abstract}
Literature review reveals that there exists, many methods for 3D visualization of a given object or a set of objects ${ }^{[1,2]}$. To name a few commonly used methods, we have Stereoscopic 3D visualization $^{[3]}$, Cross eye visualization, Parallel eye visualization, Perspective $3 \mathrm{D}$ visualization etc. An optimu location of the viewing point for an ideal 3D perspective view is attempted in this paper. The conclusions drawn are presented ${ }^{[4,5]}$.
\end{abstract}

Key words: Picture plane, color gradation, views, perspective projection, plan, elevation

\section{INTRODUCTION}

The observer's position mostly determines the clarity and visibility of the 3 mutually perpendicular dimensions/details of the object in the 3D environment ${ }^{[3]}$. The flexibility in fixing the observers position, in the case of stereoscopy, parallel and cross eye $3 \mathrm{D}$ visualization techniques is limited to human capabilities. In the case of Perspective 3D Visualization techniques, which are extensively used for Computer Generated images, the position of the observer can be varied using Computer capabilities (through suitably developed algorithms). Theoretically, we can imagine the observer moving along infinite number of paths ${ }^{[6]}$, assuming infinite number of position in each of the infinite paths.

An attempt is made in this paper to study the variation of the 3D Perspective Views, as the Observer's eye position takes different locations and to fix an optimum position for the Observer's eye for an ideal perspective view of the given 3D environment.

An example of a group of Geometric solids kept behind the picture plane (PP) is assumed. The observer moves from extreme left to extreme right along a circular path. At each of the position of the observer, the perspective $3 \mathrm{D}$ view can be generated by making use of a set of algorithms developed. The generated 3D views are closely examined to choose the optimum location of the observer for an ideal 3D perspective view of the given set of objects. A brief introduction to perspective projections is presented. The perspective elements namely the picture plane (PP), the ground plane(GP), the observer position along with the circular path to be traced by the observer are also shown.

The color gradation theory is used for giving a gray color shading on the surfaces of the $3 \mathrm{D}$ objects using the generic algorithms. The 3D perspective view of the solids generated for a few specimen locations of the observer are shown. Based on those specimen views, results are drawn and listed. The various conclusions are presented at the end.

However, clear an image of an object/group of objects, appears on the monitor, it fails to give the realistic picture with regard to depth or the third dimension ${ }^{[2]}$. This is an inherent drawback associated with all 2D images. Various techniques for 3D visualization of 2D images have been developed. Among them the Stereoscopic 3D visualization ${ }^{[4,5]}$ is popular. However, this suffers from the set back, namely a pair of colored glasses are to be used by the observer for 3D visulisation. The other techniques like parallel and cross eye 3D visualization also suffer from a similar set back of using attachments to the eyes.

Another popular technique is Perspective 3D visulaisation. While the above mentioned techniques namely Stereoscopy etc. are sued for computer acquired images (Photographs), the perspective 3D visualization method is commonly used for Computer Generated images. The perspective 3D views are generated using Computer Capabilities. The various parameters involving the perspective elements, namely the position of the object with respect to the picture plane, the dimensions of the objects, the position of the observer with respect to the picture plane and ground plane, can be stored as variables and linked in the algorithms for their wide variations. The perspetive $3 \mathrm{D}$ view is generated with a high degree of flexibility. Further, through a suitably developed algorithm a continuous movement for the observer along a chosen path will help us in fixing the optimum location of the observer/view point for an ideal 3D perspective view of a given object/set of objects. We can also think of elliptical or other types of paths for the station point/observer's position. However, in our present analysis we have considerd a circular path, since travelling through circular paths appears to be more convenient. 


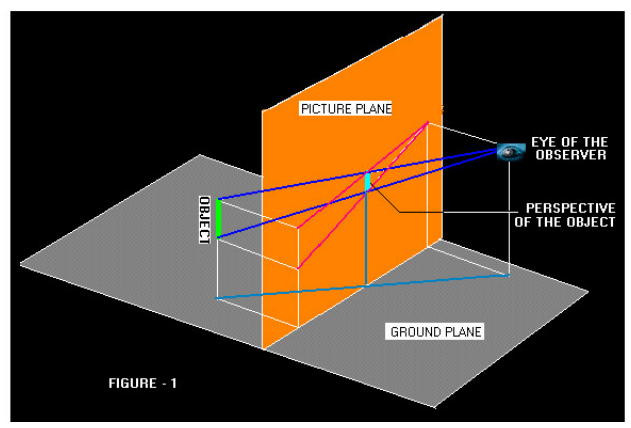

Fig. 1: Perspective view

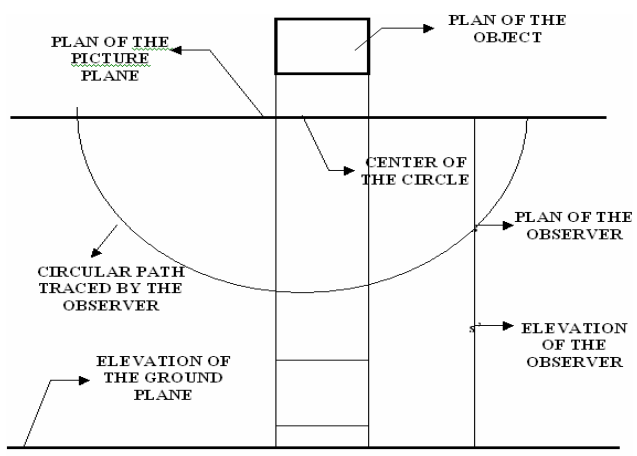

Fig. 2: Plan views of the PP

Perspective projection: Contrary to the images obtained through Orthographic projections, the images or views obtained through perspective projections are more realistic and are more amenable for processing to yield best viewing points ${ }^{[1]}$. Basically perspective projections are the images obtained on a plane called as picture plane(PP), kept perpendicular to a horizontal Group plane(GP). The object is assumed to be behind the picture plane and the observer infront of the picture plane and above Ground plane. The visual rays from the eye of the observer reaching the boundaries of the object will have to penetrate through the picture plane. These penetration points lying on the picture plane are joined by smooth curves/lines to form an image of the given object on the picture plane. This image is defined as the perspective view of the object. Figure 1 gives the various perspective elements.

Methodology for location of an optimum viewing point (an ideal 3D perspective view): A group of Geometric 3D solids are placed behind PP and above GP at different positions with respect to PP and GP. The station point/observer's position is made to move along an arc of a circle from extreme left to extreme right covering an angle of 180 . Through suitably developed algorithms the 3D perspective view is generated as the observer moves along the arc of a circle of a defined radius. For each of the position of the observer the perspective view is considered and a suitable color grading on the surfaces is given confirming to the theory of color gradation. A few positions of the observer are selected and the colored

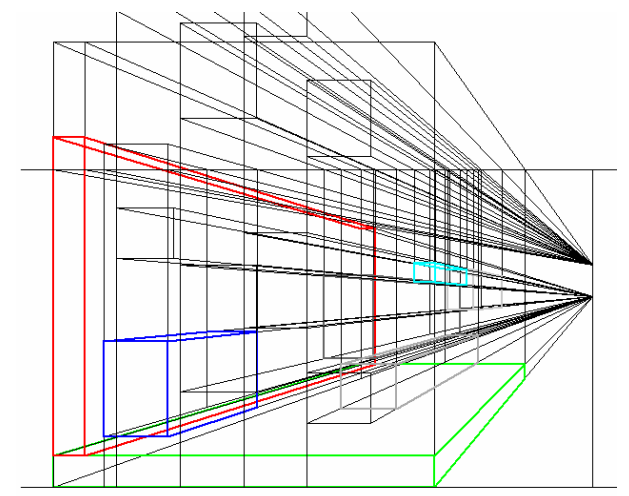

Fig. 3a: Wire frame diagram

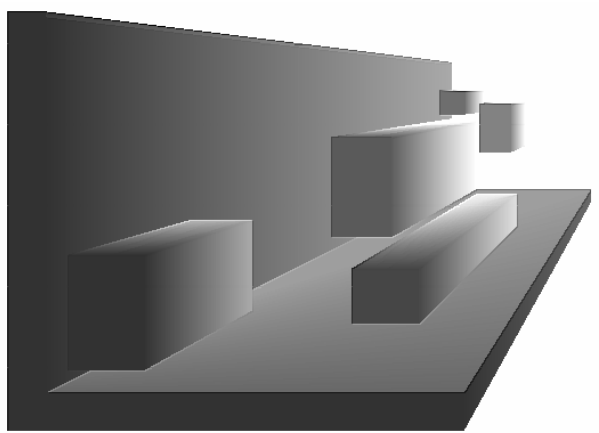

Fig. 3b: 3D perspective view

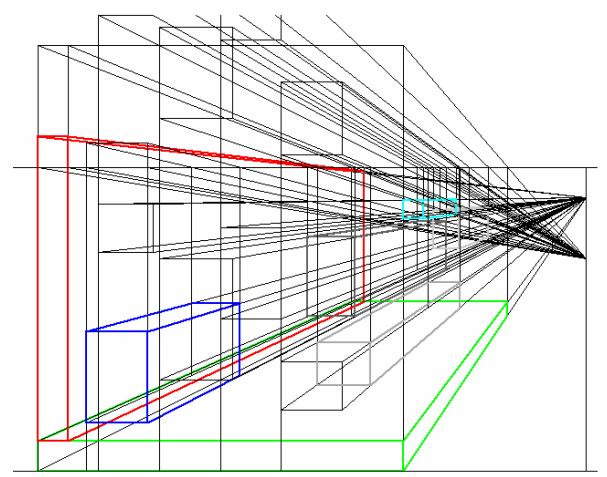

Fig. 4a: Wire frame diagram

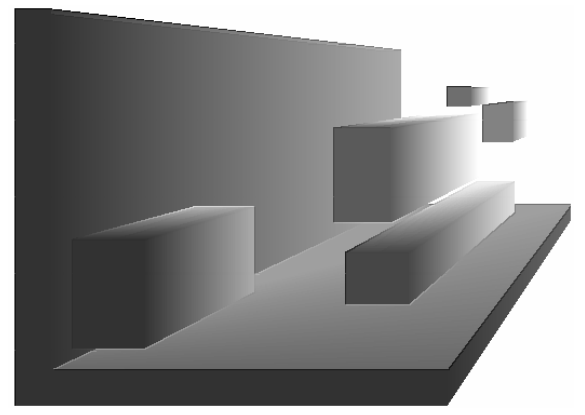

Fig. 4b: 3D perspective view

images are examined for their 3D readability. From these observed images the best image giving an ideal $3 \mathrm{D}$ effect for the environment is chosen and is fixed as the final position for 3D viewing. 


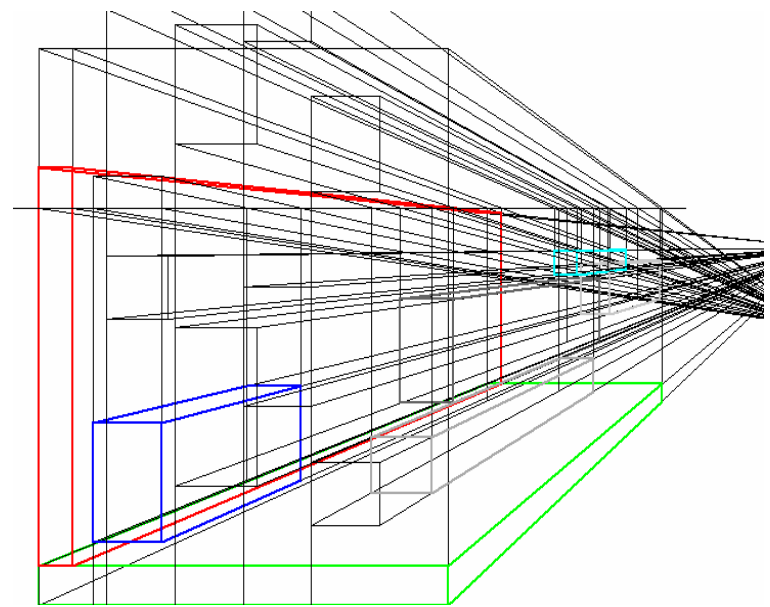

Fig. 5a: Wire frame diagram

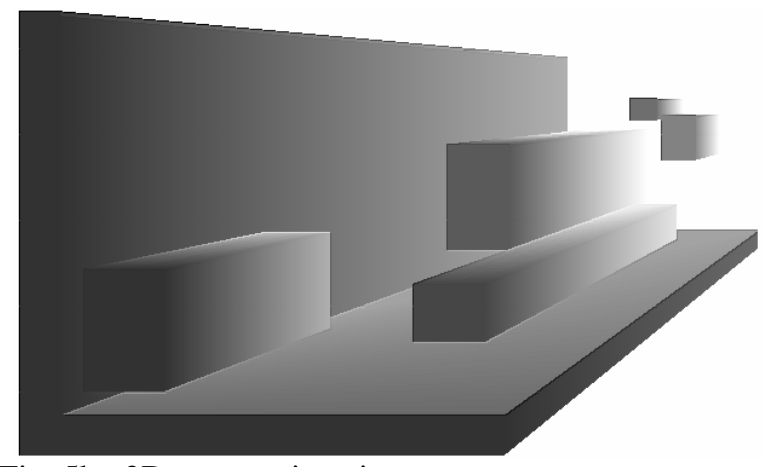

Fig. 5b: 3D perspective view

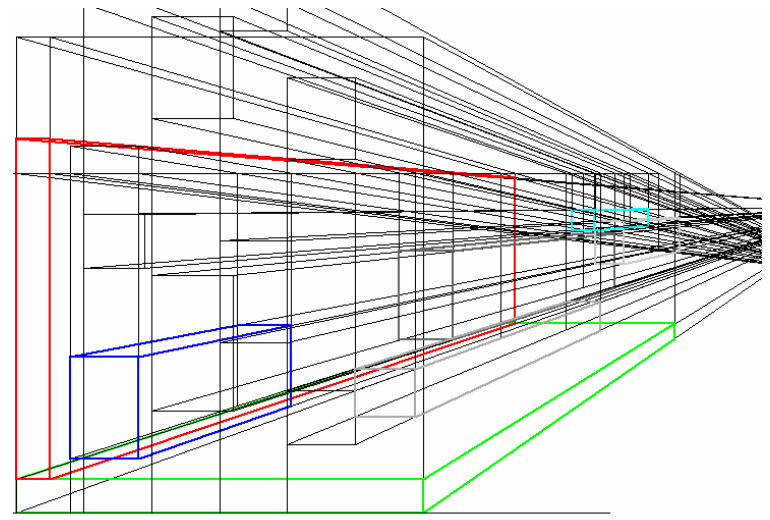

Fig. 6a: Wire frame diagram

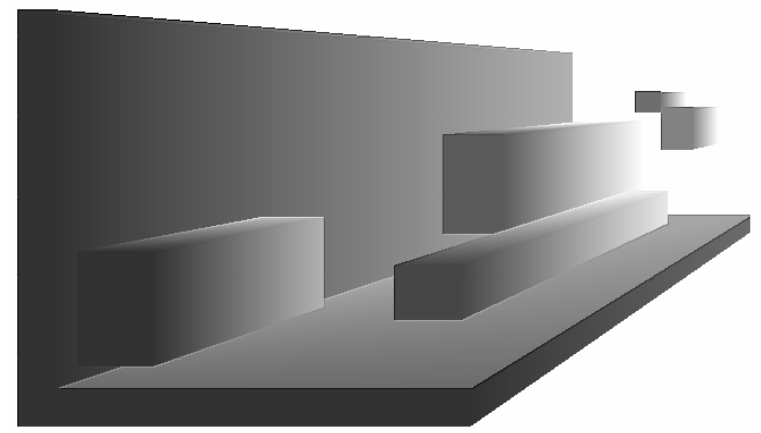

Fig. 6b: 3D perspective view
Figure 2 gives the plan views of the PP, set of objects, observes position, the path to be traced by the observer. The 3D views for a few positions of the observer is presented through Fig. 3-6. Figure 3a gives the wire frame diagram along with generated construction for the 3D perspective view. Figure $3 \mathrm{~b}$ gives the $3 \mathrm{D}$ perspective view of the solids with the color shading on different surfaces developed as per the color gradation theory.

Similar Fig. 4a, b, 5a,b and 6a,b represent the 3D views for different positions of the observer.

\author{
Generic algorithms \\ For $\mathrm{I}=\mathrm{x} 27$ To $\mathrm{x} 29$ \\ If newx $26<x 26$ Then \\ Line (newx24, newy24)-(newx26, newy26), \\ $\mathrm{RGB}(\mathrm{R}, \mathrm{R}, \mathrm{R})$ \\ newx26 = newx24 \\ newy $26=$ newy 24 \\ newx24 = newx $24+1$ \\ newy $24=$ newy $24-\tan A$ \\ Line (newx28, newy28)-(newx30, newy30), \\ $\mathrm{RGB}(\mathrm{R}, \mathrm{R}, \mathrm{R})$ \\ newx30 $=$ new 28 \\ newy $30=$ newy 28 \\ newx $28=$ newx $28+1$ \\ newy $28=$ newy $28-\tan B$ \\ newx $29=$ newx 28 \\ newy $29=$ newy 28 \\ newx32 $=$ newx $28+1$ \\ newy $32=$ newy $28-\tan C$ \\ n.......... \\ ............

\section{RESULTS AND DISCUSSION}

The ideal 3D perspective view depends on the relative positions of the object with respect to PP and GP in the 3D environment.

For a given position of the objects the ideal 3D perspective view can be identified by carefully analyzing the different views corresponding different positions of the observer.

A circular path is chosen for the position of the observer's eye since this offers easy writing down of algorithms without much complications. Once a satisfactory 3D feel of the environment is visualized then a future work in this direction can be carried out by assuming different $\mathrm{p}$ aths for the observer's movement.

\section{CONCLUSION}

The flexibility in fixing view points enables us to choose an optimum position of the observer for an ideal 3D perspective view.

With slight modifications in the algorithms used for above work, the above analysis can be effectively used for computer acquired images also. 


\section{REFERENCES}

1. Osawa, N., K. Asai and F. Saito, 2002. An interactive toolkit library for 3D applications: it3d. Eigth Eurographics Workshop on Virtual Environments, (EGVE2002), pp: 149-157.

2. Anonymous, 2002. A powerful 3D authoring method $U$ sing 2D image processing techniques 1Hiroaki Nishino : Computer Engineering \& Consulting -OITA, Ltd., Kitsuki, Oita 873-0008.

3. Hao, H.S. and R. Kumar, 2001. A global matching framework for streo computation. Proc. Intl. Conf. Computer Vision.
4. Suzuki, T., T.M. Kata and H. Tsukune, 2000. 3D object retrieval based on polygon models using rotation invariant shpe descriptors. IEEE Intl. Conf. Systems, Man and Cybernetics, Nashville, USA.

5. Ijsselsteiijn, W.A., H. de Ridder and J. Viegen, 2000. Subjective evaluation of stereoscopic images: Effects of camera parameters and display duration. IEEE Trans. Circuits and Systems for Video Technol., 10: 225-233.

6. Rueh and A. Zakhor, 2003. Constructing 3D city models by merging ground-based and airborne views. Proc. IEEE Intl. Conf. Computer Vision and Pettern Recognition. 